\title{
Benefits of the Mycorrhizal Fungi in Tomato Leaves Measured by Open Photoacoustic Cell Technique: Interpretation of the Diffusion Parameters
}

\author{
S. Sánchez-Rocha • M. Vargas-Luna • \\ G. Gutiérrez-Juárez • R. Huerta-Franco • \\ V. Olalde-Portugal
}

(C) Springer Science+Business Media, LLC 2008

\begin{abstract}
The open photoacoustic cell technique was used to measure tomato leaves from plants with and without mycorrhizal fungi (Glomus fasciculatum). Based on measurement of the photobaric contribution of the photoacoustic signal, the exponential parameter of the diffusion behavior for this contribution was calculated. From this value, the oxygen diffusion coefficient was derived. The changes in the oxygendiffusion-coefficient-dependent exponential parameter are statistically significant $(p<0.05)$ and are consistent with the expected benefits of mycorrhizal symbiosis. Potentially similar results obtained from the photothermal contribution are discussed.
\end{abstract}

Keywords Mycorrhiza - Oxygen diffusion coefficient $\cdot$ Photoacoustic $\cdot$ Thermal diffusivity · Tomato

\section{Introduction}

Mycorrhiza is a symbiotic association between plants and fungi, in which the fungi colonize the cortical tissue of roots [1]. This relationship benefits both organisms. In the case of the plant, it improves mineral uptake, particularly the uptake of phosphorous

S. Sánchez-Rocha · V. Olalde-Portugal

CINVESTAV Unidad Irapuato, Km 9.6, Libramiento Norte, Carretera Irapuato-León, 36500 Irapuato, GTO, México

M. Vargas-Luna $(\bowtie) \cdot$ G. Gutiérrez-Juárez

Instituto de Física, Universidad de Guanajuato, Loma del Bosque 103, Lomas del Campestre, 37150 León, GTO, México

e-mail: mvargas@fisica.ugto.mx

R. Huerta-Franco

Instituto de Investigaciones sobre el Trabajo, Universidad de Guanajuato, Av. Eugenio Garza Sada 572,

Lomas del Campestre, 37150 León, GTO, México 
(P) $[2,3]$. The association increases the drought resistance of the plant and its photosynthetic rate, among other benefits that have been previously studied and are still under investigation [4-7]. Most of the studies regarding the symbiosis between mycorrhizal fungi and plants have been performed using conventional techniques (e.g., gas exchange techniques such as infrared gas analysis (IRGA) [8]).

The use of photothermal techniques, particularly the use of photoacoustic (PA) cells, for research in plants is very well known [9-12]. The conventional PA cell uses a closed chamber connected internally to a microphone. A small sample of a detached leaf is placed inside the chamber and is studied by monitoring its response when illuminated by a modulated light beam $[9,10]$. The open photoacoustic cell (OPC) technique uses the same kind of microphone; however, in OPC, the microphone is sealed at the surface of the intact, still attached leaf $[13,14]$. The modulated light beam hits the leaf in the same place where the microphone is attached. The main advantage of the OPC as opposed to the conventional PA cell for plant measurements is that the OPC can be used as an in vivo measurement technique, allowing for multiple measurements of the same leaf, and thus facilitating reproducibility studies.

In both the conventional cell and the OPC, the leaf response to the modulated light beam is detected by the microphone as a pressure change around the measured region. This response is composed of two elements: the photothermal $(\mathrm{Pt})$ response and the photobaric $(\mathrm{Pb})$ response. The $\mathrm{Pb}$ contribution consists of pressure changes due to the output of modulated oxygen from the leaf. In the case of OPC, the Pt contribution comes from the pressure changes due to the thermal fluctuations of the air in the PA chamber after the absorption of the light by the sample.

Some studies have used the OPC technique in plants with mycorrhiza, including that of Acosta-Avalos et al. [14], which considered the evolution of the PA signal amplitude as a function of time after dark adaptation. Their measurements were performed in maize plants with and without mycorrhiza. They found differences in the rising time of the $\mathrm{Pb}$ component of the signal in one variety of maize (of three considered). Recently, Sanchez-Rocha et al. [15] analyzed tomato plants using the same technique and found qualitative differences in the oxygen evolution rate between preparation with and without mycorrhiza.

In an unpublished work [16] where the leaf anatomy of strawberry plants with and without mycorrhiza was analyzed, no differences were found in the total thickness of the leaves among preparations; however, the palisade parenchyma was found to be thicker than the phloem parenchyma in the plants with mycorrhiza. The palisade parenchyma is a less porous tissue than the phloem parenchyma, and a larger palisade parenchyma allows for a higher maximum rate of photosynthesis [17].

The oxygen diffusion coefficient, also known as the oxygen permeability, is widely used in models as a parameter for understanding different physiological phenomena [18]. This parameter has been measured by the PA technique to estimate, for example, $\mathrm{CO}_{2}$ internal resistance [19].

In this study, the diffusion behavior of both $\mathrm{PA}$ contributions ( $\mathrm{Pt}$ and $\mathrm{Pb}$ ) was analyzed using the OPC technique in tomato plants with and without mycorrhiza. The signal dependent on the modulation frequency, obtained from the $\mathrm{Pt}$ and $\mathrm{Pb}$ contributions of the PA signal, was examined to identify the benefits of the mycorrhizal symbiosis to the plant. We hypothesized that the benefits of the mycorrhizal 
symbiosis could be monitored by assessing the changes in the internal composition of the leaves, through the relative values of the oxygen diffusion coefficient and thermal diffusivity in the leaves of plants with and without mycorrhizal associations.

\section{Materials and Methods}

All tomato plants were grown in a sterilized 1:1 loam soil and sand mixture under comparable environmental conditions. Two weeks after seeding, half of the plants were inoculated with Glomus fasciculatum (GF) fungi during transplantation. All the plants were watered with distilled water two to three times per week, depending on the environmental temperature and relative humidity. They were fertilized weekly with a Long Ashton nutrient solution containing 22 and 44 parts per million (ppm) of P. In this study, we evaluated the response of tomato plants grown under four different experimental conditions: (1) tomatoes with GF fungi and 22 ppm of P (TG2), (2) tomatoes with GF fungi and $44 \mathrm{ppm}$ of P (TG4), (3) tomatoes without GF fungi and $22 \mathrm{ppm}$ of P (TC2), and (4) tomatoes without GF fungi and $44 \mathrm{ppm}$ of P (TC4). At the age of 8 to 9 weeks, the plants were transported to the laboratory for measurements. Two hours before measurement, the plants were moved to an environmentally controlled cabinet (controlled temperature and luminosity), to allow adaptation of the plants to the cabinet conditions. We placed a $1 \mathrm{KW}$ high-pressure sodium lamp $50 \mathrm{~cm}$ above the top of the plants to simulate sunny noon light conditions. Each of the four treatment groups comprises six plants chosen randomly from a group of more than 20 plants of each preparation, as all plants were subsequently chosen in reproducibility tests.

In previous studies, the entire plant was kept in dark conditions during the measurements, with the exception of a $3 \mathrm{~mm}$ diameter area of illumination on the leaf area being studied $[13,14]$. It is important to note that in our work, the entire plant received full illumination while the measurements were being taken. The area of the leaf that was measured received light between 600 and $730 \mathrm{~nm}$ (modulated light), comparable in amount to that of the sun's irradiation at noon.

The experimental setup [20] (Fig. 1) comprises two types of LED light sources with minimal infrared radiation: a red light (modulated) and a white light (not modulated). They were used to saturate the photosynthetic process. We made ten holes in a $2.54 \mathrm{~cm}$ diameter stainless-steel semi-hemisphere and randomly placed the LED lights within the holes. This was done to concentrate the light around the desired region. The open half of the hemisphere was placed on top of a metal plate with a circular hole $3 \mathrm{~mm}$ in diameter cut in the center. Control of the LEDs was achieved through the lock-in amplifier, which was controlled by a PC.

The irradiation levels were $55 \mathrm{~W} \cdot \mathrm{m}^{-2}$ (for modulated red light) and $235 \mathrm{~W} \cdot \mathrm{m}^{-2}$ (for non-modulated white light). It was advantageous to use the third or fourth youngest leaf so as to have a good photosynthetic rate, but also to avoid thick leaf veins (vascular bundles) and very rough surface regions. The leaf area studied was illuminated such that its upper epidermis faced the incident light. While avoiding any damage to the leaf, it was held between two copper plates that were also used as the LED and microphone holders. As mentioned, the OPC signal generated in the leaf by the modulated red-light excitation is the result of two main contributing factors. It 


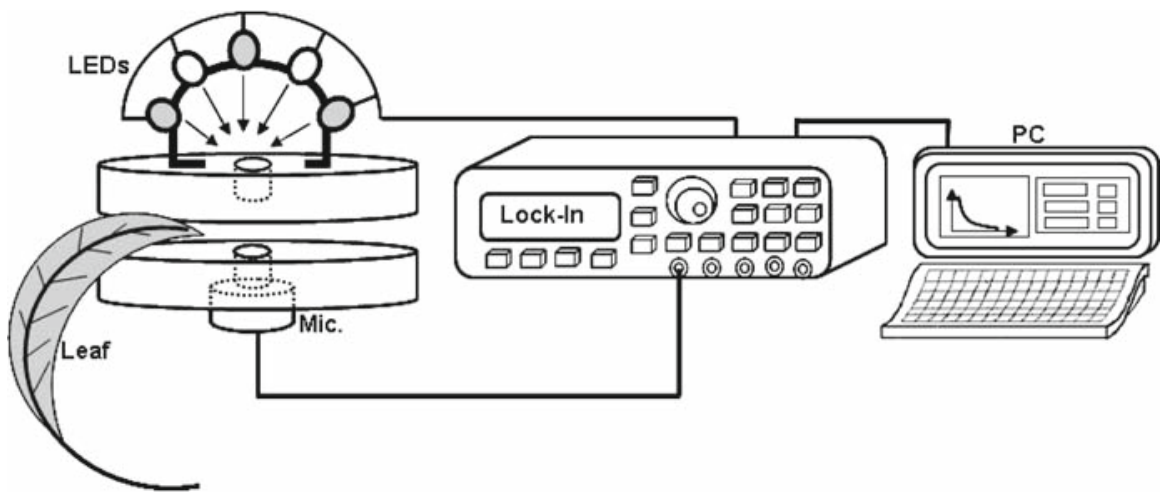

Fig. 1 Experimental setup: cross section of the LEDs holder is shown and two kinds of LEDs are indicated by white and gray ellipses (corresponding to white and red lights) which are controlled by the PC through the lock-in amplifier. Leaf holder consists of two thin copper plates

is important to note that these components are out of phase, and therefore their sum should be regarded as a vectorial quantity.

A preliminary assessment was performed on each plant to establish a baseline photoacoustic signal, so as to standardize conditions and assure that all further changes were not due to environmental changes. After reaching stable PA signal levels, we measured the PA signal as a function of frequency.

To discern both PA contributions (either in time or frequency domain), we used the following procedure. A measurement at frequencies above $200 \mathrm{~Hz}$ with modulated red light was used to estimate the Pt contribution. At these frequencies, a percentage of the red light is used for photosynthesis, but its contribution to the PA signal is dampened due to exponential attenuation. This damping is stronger than that of the Pt contribution. A second measurement using intense continuous white light was also done. This saturating continuous light satisfies the energy needs for photosynthesis, and thus almost all the modulated red light can be converted into the Pt signal (and therefore, the signal increases with saturation). Together, these two measurements give the percentage of red light used for photosynthesis (photochemical loss), which remains the same for low frequencies [21]. A third measurement is performed at low frequencies (below $200 \mathrm{~Hz}$ ) with saturated white light. Again, the energy for photosynthesis is completely supplied by the continuous white light and the percentage of red light used for photosynthesis is known, so the Pt contribution (before saturation) can be determined. The last measurement is performed, still at low frequencies without saturating continuous light, to measure the $\mathrm{Pb}$ contribution due to photosynthesis.

At low frequencies, both PA contributions were partially derived from diffusion phenomena (in addition to other Pt contributions, discussed below), which exhibit the following behavior:

$$
S=A \mathrm{e}^{-B \sqrt{f}},
$$


where $S$ is the PA signal ( $\mathrm{Pb}$ or $\mathrm{Pt}), A$ is a constant, $f$ is the modulation frequency, and $B$ is a value inversely proportional to the square root of the diffusion coefficient $(D)$ and directly proportional to a length $(\ell)$ (Eq. 2);

$$
B=\pi\left(\frac{\ell}{\sqrt{D}}\right) .
$$

In the case of the $\mathrm{Pb}$ signal, the length is the oxygen mean path through photosystem II into the stomatal chamber, within the chloroplasts in the cells of the leaf $[9,19]$. In the case of the Pt signal, $\ell$ is the total thickness of the sample. Because we are interested in the $B$ parameter, the absolute value of the signal (proportional to the $A$ parameter in Eq. 1) is non-essential.

\section{Results and Discussion}

We separated the PA signal vector into its component parts ( $\mathrm{Pt}$ and $\mathrm{Pb})$ in the frequency domain (see Fig. 2 for the amplitude). The Pb contribution to the PA signal clearly exhibits the exponential behavior mentioned above (line fitted to $\mathrm{Pb}$ in Fig. 2) when Eq. 1 is applied to the data. For the Pt signal, the general behavior does not follow that of diffusion, rather, it follows $f^{-1}$ (Fig. 3). This can be explained by thermoelastic bending, which is seen at high frequencies when there is a strong thermal gradient along the sample thickness in the thermally thick region [22]. If we look at the frequency range where the microphone responds linearly $(>10 \mathrm{~Hz})$, but before the $f^{-1}$ behavior begins (up to $30 \mathrm{~Hz}$ ), we can identify clear diffusion process behavior. In this frequency range, the thermoelastic bending contribution is comparable in magnitude to that of the thermal diffusion, but is almost frequency independent, so the general behavior of the signal resembles that of the thermal diffusion because we use behavior instead of absolute values. We estimated the photothermal $B$ parameter from this small region between 10 and $30 \mathrm{~Hz}$ (Fig. 4). Our confidence in these photothermal $B$ parameters is discussed below.

The set of $B$ values from each treatment group, considering both $\mathrm{Pb}$ and $\mathrm{Pt}$ contributions independently, does not show a normal distribution (using Shapiro-Wilk's W normality test). Therefore, a non-parametric analysis was used to look for statistical differences. Data were analyzed with a Kruskal-Wallis ANOVA by ranks' test. Differences between groups were obtained using a Mann-Whitney U test, and the results for $\mathrm{Pb}$ contributions (Fig. 5) were presented as medians, 25 and 75\% quartiles (boxes in Fig. 5), and minimum and maximum values (bars in the same figure). Statistical analysis was performed with STATISTICA, Stat-Soft, Inc., Tulsa, Oklahoma.

Statistically significant differences $(p<0.05)$ for the $B$ parameters were found between TG2 and the control samples for both P levels (TC2 and TC4) (Fig. 5). The other sample (TG4) did not show differences, due to the high variability of the data. Values of $p$ for pairs of data sets with statistically significant differences are included. Remembering that $p<0.05$ is a measure of probability, our claim that these data sets have different $B$ values has a 5\% margin of error. Similar results were found in the case of the photothermal parameter $B$ (not included in the figures). 


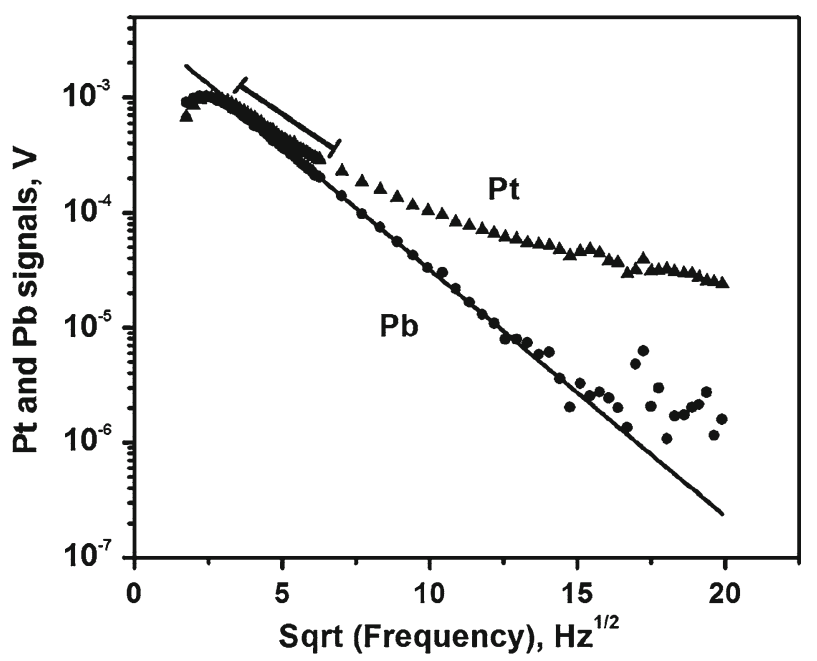

Fig. 2 Typical behavior of frequency dependence for the two PA contributions $(\mathrm{Pt}$ and $\mathrm{Pb})$. Linear response of the microphone starts at $10 \mathrm{~Hz}$. Pb contribution varies exponentially with the square root of the frequency. Pt contribution was analyzed mainly in the frequency range marked with a line above its plot

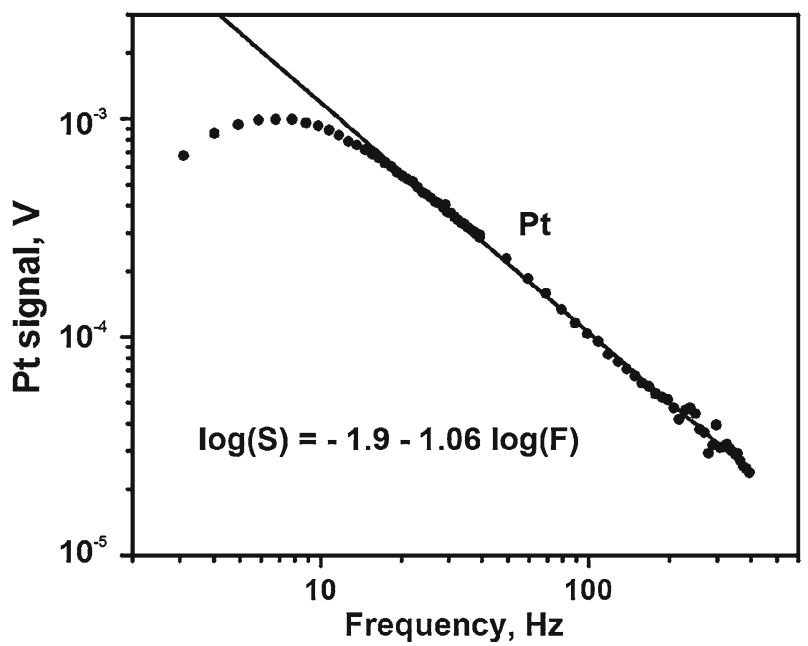

Fig. 3 Behavior of the Pt contribution: signal behaves as $f^{-1}$ due to thermoelastic bending in thermally thick samples at high frequencies

Gorton etal. [19] in 2003, working with Alocasia brisbanensis, measured the oxygen diffusion distance in palisade and spongy regions, and found it equal for both structures. We assumed that this fact is also valid in the case of the tomato plants, additionally, based on unpublished results mentioned in the introduction [16], we assumed that the palisade parenchyma structure is bigger (although the total thickness of the leaf remains the same) for tomato plants with mycorrhiza. In fact, in our study, the thickness of the leaf samples was measured in individual leaves and 


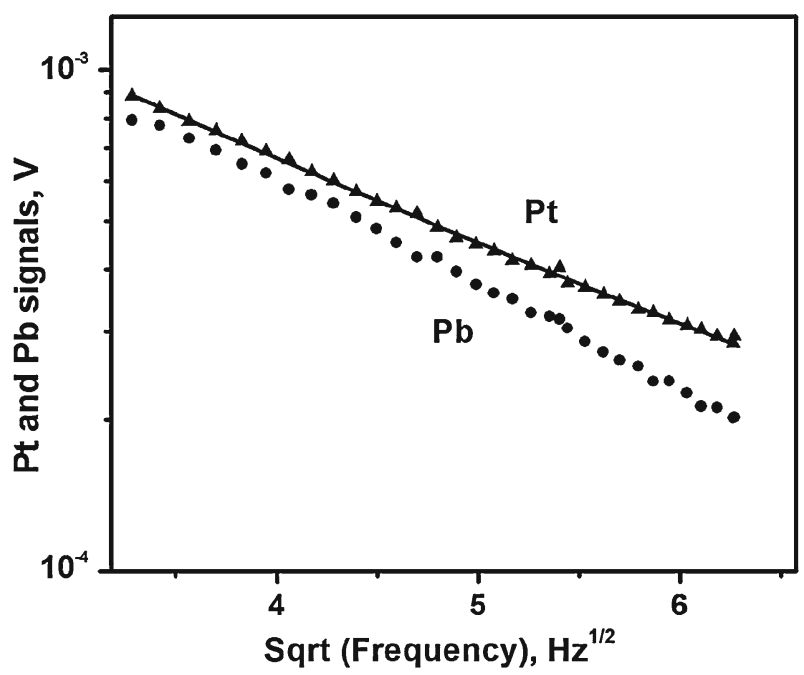

Fig. 4 Data detail at middle frequencies $(10-40 \mathrm{~Hz})$ show exponential behavior as a function of the square root of the frequency (diffusion process) for Pt contribution

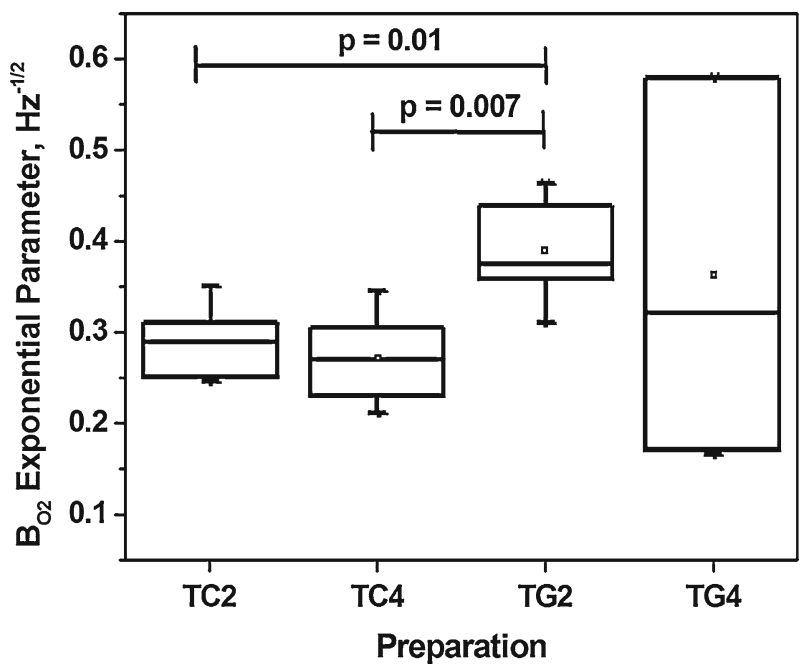

Fig. 5 Photobaric $B$ exponential parameter from tomato plants for four treatments: controls with 22 and 44 ppm of P (TC2 and TC4, respectively) and plants with GF fungi with 22 and 44 ppm of P (TG2 and TG4, respectively). The results are shown as medians, 25 and 75\% quartiles (boxes), and minimum and maximum values (bars) from samples measured in each preparation. The value $p$ denotes error probability (probability that there were no statistically significant differences between the sets of values considered)

showed non-significant differences among the different preparations (Table 1). These two assumptions are consistent with $B$ changes.

Due to the first assumption, the possible differences in the thickness of the parenchyma structure, which are due to the mycorrhiza, are inconsequential in the variation of the $B$ parameter of the $\mathrm{Pb}$ component. Therefore, $B$ would change as a result of 
Table 1 Average and standard error of leaf thickness $(\ell)$, oxygen diffusion coefficient $\left(D_{\mathrm{O}_{2}}\right)$ estimated with an oxygen mean path of $2 \mu \mathrm{m}$, and thermal diffusivity $\left(D_{\text {th }}\right)$ determined using the measured thickness of each sample

\begin{tabular}{llll}
\hline Treatment & $\begin{array}{l}\ell \pm \mathrm{SE} \\
\left(10^{-4} \mathrm{~m}\right)\end{array}$ & $\begin{array}{l}D_{\mathrm{O}_{2}} \pm \mathrm{SE}(\text { estimated }) \\
\left(10^{-10} \mathrm{~m}^{2} \cdot \mathrm{s}^{-1}\right)\end{array}$ & $\begin{array}{l}\mathrm{D}_{\text {th }} \pm \mathrm{SE} \\
\left(10^{-6} \mathrm{~m}^{2} \cdot \mathrm{s}^{-1}\right)\end{array}$ \\
\hline TG2 & $2.2 \pm 0.1$ & $0.8 \pm 0.1$ & $1.1 \pm 0.1$ \\
TG4 & $2.1 \pm 0.2$ & $1.0 \pm 0.2$ & $0.9 \pm 0.2$ \\
TC2 & $2.4 \pm 0.1$ & $1.5 \pm 0.2$ & $2.3 \pm 0.2$ \\
TC4 & $1.9 \pm 0.2$ & $1.8 \pm 0.4$ & $1.8 \pm 0.4$ \\
\hline
\end{tabular}

Preparations used were TG2 for tomatoes with GF and $22 \mathrm{ppm}$ of P, TG4 for tomatoes with GF and $44 \mathrm{ppm}$ of P, TC2: tomatoes without GF and $22 \mathrm{ppm}$ of $\mathrm{P}$, and TC4, tomatoes without GF and $44 \mathrm{ppm}$ of $\mathrm{P}$

changes in the diffusion parameter at the cellular level. If we use an oxygen mean path on the order of $\mu \mathrm{m}$ ( $2 \mu \mathrm{m}$ used in Table 1 calculations), the estimates of the oxygen diffusion coefficients $\left(D_{\mathrm{O}_{2}}\right)$ are an order of magnitude lower than that of pure water $\left(1.0 \times 10^{-9} \mathrm{~m}^{2} \cdot \mathrm{s}^{-1}\right)$. This finding resembles the results of Gorton et al. [19] in their work with Alocasia brisbanensis. More importantly, the coefficients from plants with mycorrhiza have lower values than those from plants without mycorrhiza (Table 1). According to the same authors [19], this type of difference indicates a greater viscosity at the cellular level, which gives a greater resistance to the propagation of the oxygen fluctuations.

In the case of the thermal $B$ parameter, Eq. 1 is the PA approximation for a homogeneous, isotropic, and linear sample in the thermally thick region (low frequencies). In this instance, as the thickness of the samples $(\ell)$ remains the same, all changes of $B$ are due to changes in thermal diffusivity $\left(D_{\mathrm{th}}\right)$.

The calculated values of $D_{\text {th }}$ are between those for water and air, which is consistent with the porous structure of the leaf. The plants with mycorrhiza show a thermal-diffusivity value closer to that of water (Table 1). These results show a direct relationship between the size of the palisade parenchyma (a less porous structure) and the $D_{\text {th }}$ values.

Although the results from the Pt contribution measurements seem consistent with our hypothesis, we are uncertain of the effects of the substantial thermoelastic bending occurring at low frequencies, the behavior of which was not measured. Therefore, we must consider the results of the photothermal contribution as only preliminary and perform further thermoelastic discrimination analyses.

\section{Conclusions}

The OPC technique was used to estimate the exponential parameter of the $\mathrm{Pb}$ contribution of the PA signal in tomato plants with and without mycorrhiza, based on diffusion behavior.

The estimate of the oxygen diffusion coefficient is lower in value for plants with mycorrhiza than those for plants without mycorrhiza. This result concurs with previous findings that there is an incremental difference in the viscosity of the cytosol at the cellular level in plants with mycorrhiza as opposed to those without. 
Based on the fact that the thickness of the sample does not vary with treatment, the Pt contribution demonstrates similar results in the frequency range of 10 to $30 \mathrm{~Hz}$. This is consistent with the direct relationship between the thermal diffusivity and the size of the palisade structure in plants with and without mycorrhiza; however, the strong thermoelastic bending behavior forces us to consider the results from the $\mathrm{Pt}$ contribution of the PA signal as only preliminary.

The results show that the OPC technique is highly sensitive in detecting differences among oxygen diffusion coefficients and is therefore useful in assessing internal structural differences between tomato plants with and without mycorrhiza.

Acknowledgments The authors thank Kathleen Capaccione for a review of the manuscript's English and R. Serrato, F. Alejo, J. C. Martínez, J. M. Noriega, and R. Martínez for technical support. We also thank Universidad de Guanajuato, CINVESTAV-Irapuato and CONACYT (SEP-2003-C02-44058) for financial support.

\section{References}

1. S.E. Smith, D. J. Reid, Mycorrhizal Symbiosis (Academic Press, New York, 1997)

2. E. George, K. Häussler, S.K. Kothari, X.L. Li, H. Marschner, Mycorrhizal hyphae to nutrient and water uptake of plants, in Mycorrhizas in Ecosystems, ed. by D.J. Read, D.H. Lewis, A.H. Fitter, I.J. Alexander (CAB International, Wallingford, Oxon, 1994), pp. 42-48

3. H. Marschner, Mineral Nutrition of Higher Plants (Academic Press, New York, 1998)

4. U. Drügue, F. Schönbeck, J. Plant Physiol. 141, 40 (1992)

5. D. Louche-Tessandier, G. Samson, C. Hernández-Sebastià, P. Chagvardieff, H. Desjardins, New Phytol. 142, 539 (1999)

6. G. Al-Karaki, B. McMichael, J. Zak, Mycorrhiza 14, 263 (2004)

7. R. Porcel, J.M. Ruiz-Lozano, J. Exp. Bot. 55, 1743 (2004)

8. L. Aguilera-Gómez, F.T. Davies, V. Olalde Portugal, S.A. Duray, L. Phavaphutanon, Photosynthetica 36, 441 (1999)

9. S. Malkin, O. Puchenkov, The photoacoustic effect in photosynthesis, in Life and Earth Science, ed. by A. Mandelis, P. Hess (SPIE Opt. Eng. Press, Bellingham, Washington, 1997), pp. 17-56

10. S. Malkin, M. Havaux, Photochem. Photobiol. 74, 593 (2001)

11. A.K. Rai, K.R. Reddy, J.P. Singh, Instrum. Sci. Technol. 31, 231 (2003)

12. A.K. Rai, J.P. Singh, Instrum. Sci. Technol. 31, 323 (2003)

13. A.C. Pereira, L.M. Prioli, W.J. da Silva, G.O. Neto, H. Vargas, N. Cella, J.J. Alvarado-Gil, Plant Sci. 96, 203 (1994)

14. D. Acosta-Avalos, J.J. Alvarado-Gil, H. Vargas, J. Frías-Hernández, V. Olalde-Portugal, L.C.M. Miranda, Plant Sci. 119, 183 (1996)

15. S. Sanchez-Rocha, M. Vargas-Luna, V. Olalde-Portugal, L. Madueño, G. Gutiérrez- Juárez, R. Huerta-Franco, J. Phys. IV France 125, 803 (2005)

16. N.K. Guerrero-González, Master Thesis, CINVESTAV-IPN, Unidad Irapuato México, 1999

17. W.G. Hopkins, Introduction to Plant Physiology (John Wiley \& Sons, New York, 1999)

18. P. Geigenberger, Curr. Opin. Plant Biol. 6, 247 (2003)

19. H.L. Gorton, S.K. Herbert, T.C. Vogelmann, Plant Physiol. 132, 1529 (2003)

20. M. Vargas-Luna, L. Madueño, G. Gutiérrez-Juárez, J. Bernal-Alvarado, M. Sosa, J. L. González-Solís, S. Sánchez-Rocha, V. Olalde-Portugal, J.J. Alvarado-Gil, P. Campos, Rev. Sci. Instrum. 74, 706 (2003)

21. M.H. de Paula, C.A. Vinha, M.A. Cortez, S. Placencia, Biochim. Biophys. Acta 1321, 179 (1997)

22. G. Rousset, F. Lepoutre, L. Bertrand, J. Appl. Phys. 54, 2383 (1983) 